\title{
Comparison of Sacubitril/Valsartan Versus Enalapril in the Management of Heart Failure
}

Shehar Bano ${ }^{1}$, Pooja Bai ${ }^{2}$, Sameet Kumar ${ }^{3}$, Nomesh Kumar ${ }^{4}$, Ahmed Ali ${ }^{5}$, FNU Pariya ${ }^{6}$, FNU Versha ${ }^{2}$, Dua Khalid ${ }^{6}$, Haya Khalid ${ }^{6}$, Amber Rizwan ${ }^{7}$

1. Internal Medicine, University of Health Sciences, Lahore, PAK 2. Internal Medicine, Liaquat University of Medical and Health Sciences, Jamshoro, PAK 3. Internal Medicine, Chandka Medical College Larkana, Karachi, PAK 4. Internal medicine, Liaquat University of Medical and Health Sciences, Jamshoro, PAK 5. Infectious Diseases, University of Louisville, Louisville, USA 6. Internal Medicine, Jinnah Sindh Medical University, Karachi, PAK 7. Family Medicine, Jinnah Postgraduate Medical Center, Karachi, PAK

Corresponding author: Shehar Bano, sheharbano2609@gmail.com

\section{Abstract}

\section{Background and objective}

The recent emergence of new molecules like angiotensin receptor-neprilysin inhibitor (ARNI) has highlighted the need for an update in heart failure (HF) management, as they have proven to yield better patient outcomes compared to the traditional angiotensin-converting enzyme inhibitor/angiotensin II receptor blocker (ACEI/ARB) use. This study aimed to compare HF-related hospitalization and death in patients on either ACEI/ARBs or ARNI in a local setting.

\section{Methods}

This two-arm interventional study was conducted in the cardiology and internal medicine units of a tertiary care hospital in Pakistan from July 2018 to December 2020. After enrollment, participants were randomized into two groups as per 1:1 ratio using an online research randomizer software (https://www.randomizer.org). Group A received 24/26 or 49/51 mg sacubitril/valsartan twice daily for HF. Group B received $2.5 \mathrm{or} 5 \mathrm{mg}$ enalapril twice daily. Patients were followed up for 12 months or till the development of an event.

\section{Results}

The sacubitril/valsartan group had significantly fewer HF-related hospitalizations compared to the enalapril group (13.8\% vs. $22.4 \%$; $p$-value: 0.03 ), with a relative risk reduction (RRR) of $38.3 \%$. The sacubitril/valsartan group had $52 \%$ RRR for HF-related deaths compared to the enalapril group.

\section{Conclusion}

Based on our findings, treatment with sacubitril/valsartan was superior to enalapril in reducing the risk of hospitalization and death related to HF. The magnitude of the beneficial effects of sacubitril/valsartan as compared to enalapril on cardiovascular mortality was at least as high as that of long-term treatment with enalapril.

Review began 06/22/2021 Review ended 06/30/2021 Published 07/12/2021

\section{(c) Copyright 2021}

Bano et al. This is an open access article distributed under the terms of the Creative Commons Attribution License CC-BY 4.0., which permits unrestricted use, distribution, and reproduction in any medium, provided the original author and source are credited.
Categories: Cardiology, Internal Medicine

Keywords: outcome, enalapril, hear failure, hospitalization, saccubtril/vaslartan

\section{Introduction}

Heart failure (HF) is a clinical syndrome, which is characterized by the failure to maintain cardiac output necessary to meet the oxygen demands of the body due to dysfunctional cardiac muscles and neurohormonal activity [1,2]. It is a progressive condition with no cure and requires life-long management with a combination of drugs and devices to reduce morbidity. It affects approximately 40 million people worldwide and has a one-year mortality and hospitalization rate of $7.2 \%$ and $31.9 \%$, respectively $[1,3]$. The prevalence of $\mathrm{HF}$ increases after 60 years of age, particularly in those with coronary artery disease, hypertension, and other cardiac comorbidities $[4,5]$.

The most common symptoms in HF are dyspnea, orthopnea, fatigue, reduced exercise tolerance, ankle swelling, and other symptoms of fluid overload [4]. Based on the severity of these symptoms, the disease is classified into four categories according to the New York Heart Association (NYHA) functional classification, ranging from class I with no limitation to normal physical activity to class IV with severe limitations and symptoms at rest $[4,5]$.

The mainstay of medical management for HF entails the inhibition of the renin-angiotensin-aldosterone system (RAAS), sympathetic nervous system, and neprilysin [6]. The detrimental upregulation of the RAAS 
mediates the pathophysiology and progression in HF, leading to fluid retention, vasoconstriction of the peripheral arteries, hypertrophy, and remodeling of the cardiac tissue [7]. For this reason, angiotensinconverting-enzyme inhibitors (ACEIs) and angiotensin II receptor blockers (ARBs) have been recommended as the mainstays of management of the condition. Several studies have found that the inhibition of the RAAS by either ACEIs or ARBs considerably reduces morbidity and mortality in HF [8-11].

The recent indication of the role of angiotensin receptor-neprilysin inhibitor (ARNI) has highlighted the need for an update in HF management. Neprilysin is a proteolytic enzyme that breaks down several peptides essential for the regulation of renal and cardiovascular metabolism and homeostasis. Inhibition of this pathway by ARNI has proven to yield better patient outcomes as compared to the traditional ACEI/ARB use $[6,12]$. The Prospective Comparison of ARNI with ACEI to Determine Impact on Global Mortality and Morbidity in Heart Failure (PARADIGM-HF) trial, which compared the effects of enalapril (ACEI) and sacubitril/valsartan (ARNI) on chronic HF patients, found that compared to enalapril, sacubitril/valsartan showed a better reduction in cardiovascular mortality and HF-related hospitalizations in patients with chronic HF $[6,13]$.

There is no local data available comparing the efficacy of conventional methods with that of new treatment options for HF. In light of that, the objective of this study was to compare HF-related hospitalization and death in patients on either ACEI/ARBs or ARNI in a local setting.

\section{Materials And Methods}

This longitudinal study was conducted in the cardiology and internal medicine units of a tertiary care hospital in Pakistan from July 2018 to December 2020. The ethical review board approval was obtained before the enrollment of patients. After obtaining informed consent, 400 treatment-naïve patients with HF were enrolled in the study via consecutive convenient non-probability sampling. Diagnosis of HF was made based on the clinical presentation of shortness of breath, weakness, and edema, reduced left ventricular ejection fraction (<40\%), and elevated N-terminal pro-B-type natriuretic peptide (NT-proBNP). The cut-off value for NT-proBNP for patients aged $<50$ years was $450 \mathrm{pg} / \mathrm{mL}$, and it was $900 \mathrm{pg} / \mathrm{mL}$ for patients $\geqslant 50$ years in age [14].

After enrollment, the participants were randomized into two groups as per 1:1 ratio using an online research randomizer software (https://www.randomizer.org). Group A received 24/26 or 49/51 mg sacubitril/valsartan twice daily for HF. Group B received 2.5 or $5 \mathrm{mg}$ enalapril twice daily. The decision on dosing was made based on the severity of the symptoms.

The patients' characteristics such as age, gender, history of smoking, blood pressure, previous history of myocardial infarction, ejection fraction, and NT-proBNP were recorded in a self-structured questionnaire. Patients were followed up for 12 months or till the development of an event. The event was defined as hospitalization or death related to HF.

The number of participants lost to follow-up in the sacubitril/valsartan and enalapril group was 19 and 17, respectively. The final analysis included only those participants who completed the study. Statistical analysis was performed using the SPSS Statistics version 22.0 (IBM, Armonk, NY). Continuous variables were analyzed via descriptive statistics and were presented as mean and standard deviation (SD). Categorical variables were presented as percentages and frequencies. Relative risk (RR) and relative risk reduction (RRR) were calculated via an online calculator (MedCalc) using a 95\% confidence interval. A p-value of less than 0.05 meant that there is a significant difference between the two groups and the null hypothesis is void.

\section{Results}

The characteristics between both groups were comparable, indicating that our randomization was successful (Table 1). 


\section{Cureus}

\begin{tabular}{|c|c|c|c|}
\hline Characteristics & Sacubitril/valsartan group $(n=181)$ & Enalapril group $(n=183)$ & P-value \\
\hline Age in years $($ mean $\pm S D)$ & $53 \pm 12$ & $55 \pm 12$ & 0.12 \\
\hline Male, $n(\%)$ & $88(48.62 \%)$ & $90(49.18 \%)$ & 0.91 \\
\hline Hypertension, n (\%) & $165(91.16 \%)$ & 170 (92.90\%) & 0.54 \\
\hline Smoking, n (\%) & $52(28.73 \%)$ & $55(30.05 \%)$ & 0.78 \\
\hline Diabetes, n (\%) & $68(37.57 \%)$ & $65(35.53 \%)$ & 0.68 \\
\hline Hypercholesterolemia, n (\%) & $100(55.25 \%)$ & 106 (57.92\%) & 0.60 \\
\hline $\mathrm{BMI}$ greater than $25 \mathrm{~kg} / \mathrm{m}^{2}, \mathrm{n}(\%)$ & $56(30.94 \%)$ & $50(27.32 \%)$ & 0.44 \\
\hline Ejection fraction, \%, (mean $\pm S D$ ) & $31.21 \pm 4.12$ & $31.82 \pm 4.02$ & 0.10 \\
\hline \multicolumn{4}{|l|}{ NT-proBNP (pg/mL), (mean \pm SD) } \\
\hline Age below 50 years & $542.12 \pm 50.12$ & $551.22 \pm 48.52$ & 0.07 \\
\hline Age 50 years and above & $1201.23 \pm 101.34$ & $1211.61 \pm 99.98$ & 0.32 \\
\hline
\end{tabular}

\section{TABLE 1: Characteristics of the participants}

SD: standard deviation; BMI: body mass index; NT-proBNP: N-terminal pro-B-type natriuretic peptide

The sacubitril/valsartan group had significantly fewer HF-related hospitalization compared to the enalapril group (13.8\% vs. $22.4 \%$; p-value: 0.03 ). The RRR was $38.3 \%$. The sacubitril/valsartan group had a $52 \%$ RRR

for HF-related death compared to the enalapril group; however, the difference was not significant (Table 2).

\begin{tabular}{|c|c|c|c|c|c|c|}
\hline Events & $\begin{array}{l}\text { Sacubitril/valsartan group, } n(\%) \text {, } \\
(n=181)\end{array}$ & $\begin{array}{l}\text { Enalapril group, } n(\%) \text {, } \\
(n=183)\end{array}$ & RR (95\% CI) & $\begin{array}{l}\text { RRR } \\
\text { (\%) }\end{array}$ & $\begin{array}{l}\text { Number needed to } \\
\text { treat }\end{array}$ & $\begin{array}{l}\text { P- } \\
\text { value }\end{array}$ \\
\hline $\begin{array}{l}\text { HF-related } \\
\text { hospitalization }\end{array}$ & $25(13.8 \%)$ & $41(22.4 \%)$ & $\begin{array}{l}0.61(0.39- \\
0.97)\end{array}$ & $38.3 \%$ & 11.63 & 0.03 \\
\hline HF-related death & $7(3.8 \%)$ & 15 (8.1\%) & $\begin{array}{l}0.47(0.19- \\
1.12)\end{array}$ & $52 \%$ & 23.1 & 0.09 \\
\hline
\end{tabular}

TABLE 2: Comparison of HF-related events between sacubitril/valsartan and enalapril groups

HF: heart failure; RR: relative risk; RRR: relative risk reduction; $\mathrm{Cl}$ : confidence interval

\section{Discussion}

The findings of our study demonstrated that sacubitril/valsartan yielded superior results as compared to enalapril in treating HF. Results of our study are consistent with other studies as similar superiority has been shown by several other studies. McMurray et al. have concluded that the inhibition of angiotensin II receptors with sacubitril/valsartan was comparatively more effective than that with enalapril in terms of RR of cardiovascular causes and hospitalization for HF [13].

Neprilysin, a neutral endopeptidase, degrades several endogenous vasoactive peptides, including the natriuretic peptides, bradykinin, and adrenomedullin [15]. Increased levels of these substances secondary to neprilysin inhibition cause neurohormonal over-activation, resulting in vasoconstriction, sodium retention, and maladaptive remodeling [16,17]. Combined inhibition of the RAAS and neprilysin has shown better results, but the clinical trials have shown serious angioedema after combined inhibition of ACEI and neprilysin [18]. Sacubitril/valsartan, consisting of neprilysin and ARB inhibitor, decreases the risk of angioedema. It is superior to enalapril in reducing the risk of mortality from any cause and also in terms of physical limitations of HF [13]. Treatment with sacubitril/valsartan led to symptomatic hypotension secondary to its vasodilator effects; however, no increase in the rate of discontinuation was observed due to this adverse effect. Impaired renal perfusion, increased serum creatinine level, and drug discontinuation due to renal impairment were more commonly seen in the enalapril group than the sacubitril/valsartan group 
[13]. The mainstay of HF therapy includes prevention of the worsening of HF and maintaining clinical stability. The mortality benefits with sacubitril/valsartan were greater as compared to enalapril. Compared with enalapril, the risk of hospitalization for HF and overall hospitalization for any cause with sacubitril/valsartan was reduced by $23 \%$ and $16 \%$, respectively [19]. Valsartan has $60 \%$ greater bioavailability when administered as sacubitril/valsartan than as a single-agent formulation. Thus, the $103 \mathrm{mg}$ of valsartan present in a $200 \mathrm{mg}$ dose of sacubitril/valsartan provides an equivalent systemic exposure to the $160 \mathrm{mg}$ dose of valsartan, which is the maximum twice-daily recommended dose for the treatment of HF [19].

To the best of our knowledge, this is the first study in a local setting on the role of sacubitril/valsartan in reducing HF-related complications and death. However, since the study was conducted at a single institute, the sample was less diverse and limited. The impact of other parameters like echo findings and electrocardiogram changes could not be assessed due to limited resources.

\section{Conclusions}

As per our findings, treatment with sacubitril/valsartan was superior to enalapril in reducing the risk of mortality and hospitalization secondary to HF. The magnitude of the beneficial effects of sacubitril/valsartan as compared to enalapril on cardiovascular mortality was at least as high as that of long-term treatment with enalapril. However, further large-scale multi-centric studies are needed to confirm the results of our study.

\section{Additional Information \\ Disclosures}

Human subjects: Consent was obtained or waived by all participants in this study. Liaquat University of Medical and Health Sciences IRB issued approval LUMHS/2018/IRB/06-11 (Version 2.0). This study has been approved by the Liaquat University of Medical and Health Sciences IRB. Animal subjects: All authors have confirmed that this study did not involve animal subjects or tissue. Conflicts of interest: In compliance with the ICMJE uniform disclosure form, all authors declare the following: Payment/services info: All authors have declared that no financial support was received from any organization for the submitted work. Financial relationships: All authors have declared that they have no financial relationships at present or within the previous three years with any organizations that might have an interest in the submitted work. Other relationships: All authors have declared that there are no other relationships or activities that could appear to have influenced the submitted work.

\section{References}

1. Maggioni AP, Dahlström U, Filippatos G, et al.: EURObservational Research Programme: regional differences and 1-year follow-up results of the Heart Failure Pilot Survey (ESC-HF Pilot). Eur J Heart Fail. 2013, 15:80817. 10.1093/eurjhf/hft050

2. Yancy CW, Jessup M, Bozkurt B, et al.: 2017 ACC/AHA/HFSA Focused Update of the 2013 ACCF/AHA Guideline for the Management of Heart Failure: a report of the American College of Cardiology/American Heart Association Task Force on Clinical Practice Guidelines and the Heart Failure Society of America. Circulation. 2017, 136:e137-61. 10.1161/CIR.0000000000000509

3. GBD 2015 Disease and Injury Incidence and Prevalence Collaborators: Global, regional, and national incidence, prevalence, and years lived with disability for 310 diseases and injuries, 1990-2015: a systematic analysis for the Global Burden of Disease Study 2015. Lancet. 2016, 388:1545-602. 10.1016/S01406736(16)31678-6

4. Chronic heart failure: National Clinical Guideline for Diagnosis and Management in Primary and Secondary Care. (2010). Accessed: May 30, 2021: https://pubmed.ncbi.nlm.nih.gov/22741186/.

5. McMurray JJ, Pfeffer MA: Heart failure. Lancet. 2005, 365:1877-89. 10.1016/S0140-6736(05)66621-4

6. Murphy SP, Ibrahim NE, Januzzi JL Jr: Heart failure with reduced ejection fraction: a review . JAMA. 2020, 324:488-504. 10.1001/jama.2020.10262

7. Hartupee J, Mann DL: Neurohormonal activation in heart failure with reduced ejection fraction . Nat Rev Cardiol. 2017, 14:30-8. 10.1038/nrcardio.2016.163

8. Cohn JN, Tognoni G: A randomized trial of the angiotensin-receptor blocker valsartan in chronic heart failure. N Engl J Med. 2001, 345:1667-75. 10.1056/NEJMoa010713

9. CONSENSUS Trial Study Group: Effects of enalapril on mortality in severe congestive heart failure. Results of the Cooperative North Scandinavian Enalapril Survival Study (CONSENSUS). N Engl J Med. 1987, 316:1429-35. 10.1056/NEJM198706043162301

10. Garg R, Yusuf S: Overview of randomized trials of angiotensin-converting enzyme inhibitors on mortality and morbidity in patients with heart failure. Collaborative Group on ACE Inhibitor Trials. JAMA. 1995, 273:1450-6.

11. Granger CB, McMurray JJ, Yusuf S, et al.: Effects of candesartan in patients with chronic heart failure and reduced left-ventricular systolic function intolerant to angiotensin-converting-enzyme inhibitors: the CHARM-Alternative trial. Lancet. 2003, 362:772-6. 10.1016/S0140-6736(03)14284-5

12. Vasquez N, Carter S, Grodin JL: Angiotensin receptor-neprilysin inhibitors and the natriuretic peptide axis . Curr Heart Fail Rep. 2020, 17:67-76. 10.1007/s11897-020-00458-y

13. McMurray JJ, Packer M, Desai AS, et al.: Angiotensin-neprilysin inhibition versus enalapril in heart failure . N Engl J Med. 2014, 371:993-1004. 10.1056/NEJMoa1409077

14. Tung RH, Camargo CA Jr, Krauser D, Anwaruddin S, Baggish A, Chen A, Januzzi JL Jr: Amino-terminal probrain natriuretic peptide for the diagnosis of acute heart failure in patients with previous obstructive 


\section{Cureus}

airway disease. Ann Emerg Med. 2006, 48:66-74. 10.1016/j.annemergmed.2005.12.022

15. Wilkinson IB, McEniery CM, Bongaerts KH, MacCallum H, Webb DJ, Cockcroft JR: Adrenomedullin (ADM) in the human forearm vascular bed: effect of neutral endopeptidase inhibition and comparison with proadrenomedullin NH2-terminal 20 peptide (PAMP). Br J Clin Pharmacol. 2001, 52:159-64. 10.1046/j.03065251.2001.1420.x

16. Maric C, Zheng W, Walther T: Interactions between angiotensin 11 and atrial natriuretic peptide in renomedullary interstitial cells: the role of neutral endopeptidase. Nephron Physiol. 2006, 103:p149-56. 10.1159/000092457

17. Kuhn M: Molecular physiology of natriuretic peptide signalling. Basic Res Cardiol. 2004, 99:76-82. 10.1007/s00395-004-0460-0

18. Rademaker MT, Charles CJ, Espiner EA, Nicholls MG, Richards AM, Kosoglou T: Combined neutral endopeptidase and angiotensin-converting enzyme inhibition in heart failure: role of natriuretic peptides and angiotensin II. J Cardiovasc Pharmacol. 1998, 31:116-25. 10.1097/00005344-199801000-00017

19. Khder Y, Shi V, McMurray JJV, Lefkowitz MP: Sacubitril/valsartan (LCZ696) in heart failure . Handb Exp Pharmacol. 2017, 243:133-65. 10.1007/164_2016_77 